\title{
Synthesis and Electronic Behavior of a Net-Worked Scandium-Phenylene-Yttrium Ternary Hybrid Copolymer
}

\author{
Hideo Matsui, ${ }^{1}$ Tetsuro Kawahara, ${ }^{2}$ Masaru Mishima, ${ }^{1}$ and Masakuni Yoshinara ${ }^{1,3, \dagger}$ \\ ${ }^{1}$ Department of Applied Chemistry, Faculty of Science and Engineering, Kinki University, \\ 3-4-1, Kowakae, Higashiosaka 577-8502, Japan \\ ${ }^{2}$ Nippon Sheet Glass Co. Ltd., 2-chome, Kaigan, Minato-ku, Tokyo 105-8552, Japan \\ ${ }^{3}$ Molecular Engineering Institute, Kinki University, 3-4-1, Kowakae, Higashiosaka 577-8502, Japan
}

(Received September 6, 2004; Accepted May 30, 2005; Published September 15, 2005)

\begin{abstract}
A ternary, scandium-phenylene-yttrium hybrid copolymer was synthesized by initially reacting 1,4hydroquinone with scandium isopropoxide below $-45^{\circ} \mathrm{C}$ followed by a second reaction with yttrium isopropoxide at room temperature. ESR spectral examinations and ab-initio calculations indicate that an electron transfer takes place initially from the phenylene group to the yttrium atom at a lower energy-level and then to the scandium atom at a higher energy-level, i.e. via two-step electron transportation. [DOI 10.1295/polymj.37.656]

KEY WORDS Organic-metal Hybrid Copolymer / Scandium / Yttrium / Hydroquinone / Two-step Electron Transportation /
\end{abstract}

Organic-inorganic hybrid materials have been expected to exhibit novel properties, and many works on the syntheses of coordinated organic-inorganic hybrid copolymers have been reported. ${ }^{1,2}$ We have considered that organic-inorganic hybrid copolymers with covalent bonding will cause unique electronic properties, because a covalent-bonded electron interaction between an organic moiety and a metal atom may be essentially different from a coordinationbonded electron interaction. In previous papers on binary hybrid copolymers having metal-organic units, an electron transfer was shown to take place from the organic moieties to such metal atoms as $\mathrm{Ti}, \mathrm{Zr}, \mathrm{Y}$, and $\mathrm{Ta}$, and a higher degree of the electron transfer was detected when using an aryl group as the organic moiety. ${ }^{3-6}$

We have assumed that, when an organic moiety is sandwiched with two different kinds of metals, a new type of hybrid copolymer with an unique electronic behavior will be given. In this work, we wish to report the synthesis and electronic behavior of a net-worked, covalent-bonded scandium-phenyleneyttrium ternary hybrid copolymer III (Scheme 1). The third ionization potential of scandium is known to be higher than that of yttrium, and thus, the combination of these metals in the copolymer is expected to cause an electron transportation to a rather high electron-acceptable scandium atom with assistance of yttrium atom. Scandium compounds are used as a photo-device and a metal-halide lump, and yttrium compounds are widely used as a fluorescent material, a laser material, a permanent magnet, and a high-temperature superconductor. Therefore, the combination

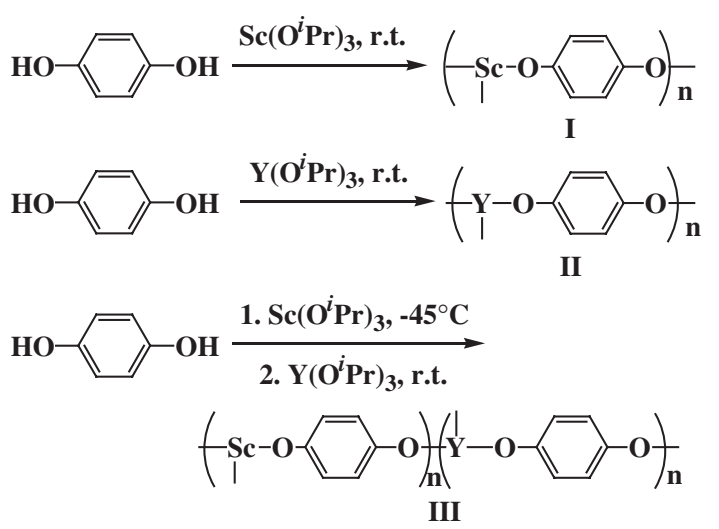

Scheme 1. Syntheses of copolymer I (Sc), II (Y), III (Sc-Y).

of these metals is also expected to cause an unique photochemical and/or electrochemical nature.

\section{EXPERIMENTAL}

\section{Reagents}

Toluene solution of yttrium isopropoxide was supplied from AZmax Co., Ltd. Scandium isopropoxide was obtained from Aldrich Co., Ltd. Other reagents and solvents were purified by the usual procedures.

\section{Syntheses of Copolymers}

A solution of scandium isopropoxide $(1 \mathrm{mmol})$ in anhydrous THF $(20 \mathrm{~mL})$ was added to a solution of 1,4-hydroquinone $(1.5 \mathrm{mmol})$ in anhydrous THF (50 $\mathrm{mL}$ ) at $-80^{\circ} \mathrm{C}$, and the mixture was stirred at room temperature overnight. Precipitate formed was collected, washed with anhydrous THF using a Soxhlet

${ }^{\dagger}$ To whom correspondence should be addressed (Tel: +81-6-6721-2332, Fax: +81-6-6727-4301, E-mail: m-yoshi@apch.kindai.ac.jp). 
extractor, and dried at $60{ }^{\circ} \mathrm{C}$ under vacuo to obtain a scandium-phenylene binary hybrid copolymer I (green-colored). Similar treatment of yttrium isopropoxide $(1 \mathrm{mmol})$ with 1 ,4-hydroquinone $(1.5 \mathrm{mmol})$ gave a yttrium-phenylene binary hybrid copolymer II (black-colored). A solution of scandium isopropoxide $(1 \mathrm{mmol})$ in anhydrous THF $(20 \mathrm{~mL})$ was added to a solution of 1,4-hydroquinone $(3 \mathrm{mmol})$ in anhydrous THF $(50 \mathrm{~mL})$ at $-60^{\circ} \mathrm{C}$, and the temperature of the mixture was raised to $-45^{\circ} \mathrm{C}$, and, then, into the mixture was added a solution of yttrium isopropoxide $(1 \mathrm{mmol})$ in anhydrous THF $(20 \mathrm{~mL})$ at $-45^{\circ} \mathrm{C}$. The reaction mixture was stirred at room temperature overnight. Precipitate formed was washed with anhydrous THF by using a Soxhlet extractor and dried at $60^{\circ} \mathrm{C}$ under vacuo to obtain a scandium-phenyleneyttirum ternary hybrid copolymer III (gray-colored).

\section{Apparatus}

Elemental analyses were performed for $\mathrm{C}$ and $\mathrm{H}$ with Yanaco MT-6 and for Sc and Y with Shimadzu ICPS-1000 III. HPDEC ${ }^{13} \mathrm{C}$ NMR spectra were taken using Bruker AVANCE-DSX400. UV-vis spectra of the copolymers were measured using Hitachi U-4000. Electron spin resonance (ESR) spectra were taken using JEOL TE-200. Transmission emission microscopy (TEM) was observed with JEOL JEM3010 spectrometer. SEM-EDX observations were done with Hitachi S-300. The mapping of the particles was taken using a SEM, Hitachi S-4800.

\section{RESULTS AND DISCUSSION}

In order to determine a procedure for synthesizing ternary hybrid copolymer III, the reactivities of scandium isopropoxide and yttrium isopropoxide with 1,4hydroquinone were examined. Scandium isopropoxide was found to react with 1,4-hydroquinone above

Table I. IR spectra of copolymers

\begin{tabular}{cc}
\hline Copolymer & Wave number $\left(\mathrm{cm}^{-1}\right)$ \\
\hline I & $1547,1502(v: \mathrm{C}=\mathrm{C}) 834(\delta: \mathrm{C}-\mathrm{H})$ \\
II & $1539,1514(v: \mathrm{C}=\mathrm{C}) 830(\delta: \mathrm{C}-\mathrm{H})$ \\
III & $1541,1503(v: \mathrm{C}=\mathrm{C}) 834(\delta: \mathrm{C}-\mathrm{H})$ \\
\hline
\end{tabular}

$-40{ }^{\circ} \mathrm{C}$ to give a precipitated scandium-phenylene binary hybrid copolymer I, but no precipitate was formed below $-45^{\circ} \mathrm{C}$. On the other hand, yttrium isopropoxide reacted with 1,4-hydroquinone even at $-80^{\circ} \mathrm{C}$ to give a yttrium-phenylene binary hybrid copolymer II. These findings suggest that the reactivity of 1,4-hydroquinone with alkoxides can be controlled by reaction temperature. Thus, by assuming that the reaction of scandium isopropoxide with 1,4-hydroquinone below $-40^{\circ} \mathrm{C}$ may produce an initial adduct, the preparation of a scandium-phenylene-yttrium ternary hybrid copolymer III was attempted in the following way. Scandium isoproxide was allowed to react initially with 1,4 -hydroquinone at $-45^{\circ} \mathrm{C}$, and, then, into the mixture was added dropwise a solution of yttrium isopropoxide at $-45^{\circ} \mathrm{C}$, and the resulting mixture was stirred at room temperature for $24 \mathrm{~h}$ to obtain a precipitated product.

The formations and the compositions of the copolymers were examined. First, the IR spectra of the copolymers were taken to show frequencies due to the phenylene group (Table I). ICP and elemental analyses were performed, and the results were summarized in Table II. Observed values of metal and carbon atoms were found to be rather lower than the calculated values. As a possible reason, an incomplete substitution reaction is considered to take place. The HPDEC ${ }^{13} \mathrm{C}$ NMR spectra of the copolymer III (ScY) show a peak due to an isopropyl group of the starting alkoxides at $25 \mathrm{ppm}$ (Figure 1), indicating that the

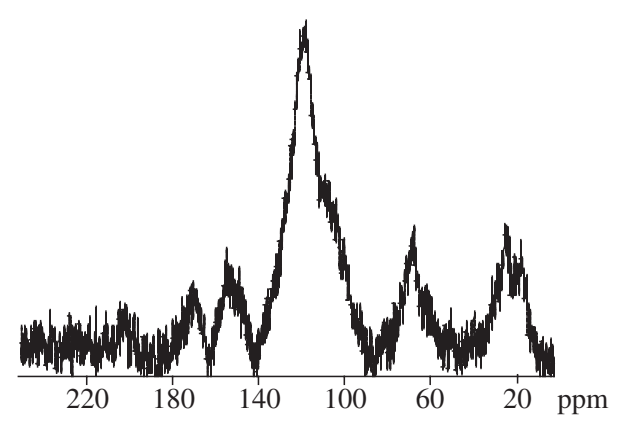

Figure 1. The HPDEC ${ }^{13} \mathrm{C}$ NMR spectrum of the copolymer III (Sc-Y). Assignment: $25 \mathrm{ppm}$ (isopropyl- $\mathrm{CH}_{3}$ ), $80 \mathrm{ppm}$ (isopropyl-CH), 120 ppm (phenylene-2,3,5,6), 150 ppm (phenylene-1,4), 180 ppm (spinning side band).

Table II. ICP and elemental analyses of copolymers

\begin{tabular}{|c|c|c|c|c|c|}
\hline \multirow{2}{*}{ Copolymer } & \multicolumn{4}{|c|}{ Found/Calcd. (\%) } & \multirow{2}{*}{$\mathrm{Sc} / \mathrm{Y}$ (ideal rate) } \\
\hline & Sc & $\mathrm{Y}$ & $\mathrm{C}$ & $\mathrm{H}$ & \\
\hline I & $16.18 / 21.71$ & - & $39.80 / 52.20$ & $4.08 / 2.82$ & \\
\hline II & - & $23.34 / 36.41$ & $29.43 / 43.06$ & $3.12 / 2.41$ & \\
\hline III & $6.18 / 9.81$ & $11.91 / 19.41$ & $40.71 / 47.19$ & $4.56 / 2.64$ & $1.03(1.00)$ \\
\hline
\end{tabular}

${ }^{\mathrm{a}} \mathrm{Sc} / \mathrm{Y}$ is the rate of metal content in the copolymer III. Sc/Y = (Found (\%) of Sc/atomic mass of Sc)/ (Found (\%) of $\mathrm{Y} /$ atomic mass of $\mathrm{Y}$ ) 

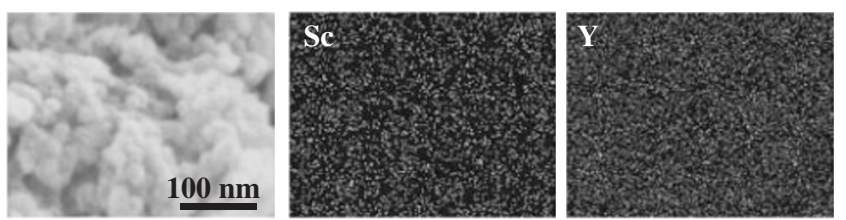

Figure 2. SEM-EDX images of the copolymer III (Sc-Y).

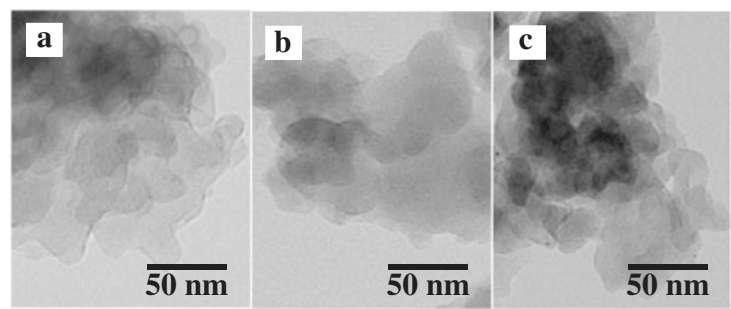

Figure 3. TEM images of the copolymers (a) copolymer I (Sc), (b) copolymer II (Y), (c) copolymer III (Sc-Y). The measurement condition: accelerating voltage $300 \mathrm{kV}$.

alkoxides insufficiently reacted with 1,4-hydroquinone. Therefore, the accurate ratio of metal content against phenylene content in the copolymers was unable to be determined. However, a Sc/Y ratio for III was determined to be 1.03 , which is very close to an expected ratio, 1. The SEM-EDX images of copolymer III (Sc-Y) showed that Sc and Y uniformly dispersed in the particles (Figure 2). From these results, we believe that phenylene-metal units are involved in the copolymers. Incidentally, the TEM images of copolymers I-III (Figure 3) showed that they were essentially amorphous particles with the diameters of $c a$. $20-50 \mathrm{~nm}$. It is noted that a TEM electron beam penetrates through the particles of the copolymers and the overlapped parts of the particles become dim, suggesting that the particles are half-transparent.

The colors of the copolymers were interestingly found to vary by changing the metals, and their UV-vis spectra were thus taken (Figure 4). All the copolymers were shown to have the absorption bands over the entire range of wavelength, indicating that the copolymers have high electron excitation abilities, and, further, the intensities were found to increase in the order of II $>$ III $>$ I. Our understanding is that an electron transfer between the phenylene group and the metal atoms occurred. The ESR spectra of copolymers I-III were measured (Figure 5). It was found that a peak due to a free electron at $337 \mathrm{mT}$ ( $\mathrm{g}=c a .2 .004)$ was observed, and that the intensities increased in the order of II $>$ III $>$ I, which agrees with the order of the UV-vis spectra. The radical spin quantities of the copolymers by the double integrating calculation of differential absorption line with the use of DPPH were determined to be $7.75 \times 10^{20} / \mathrm{g}$ for II, $5.48 \times 10^{20} / \mathrm{g}$ for III, and $3.88 \times 10^{20} / \mathrm{g}$ for I, respec-

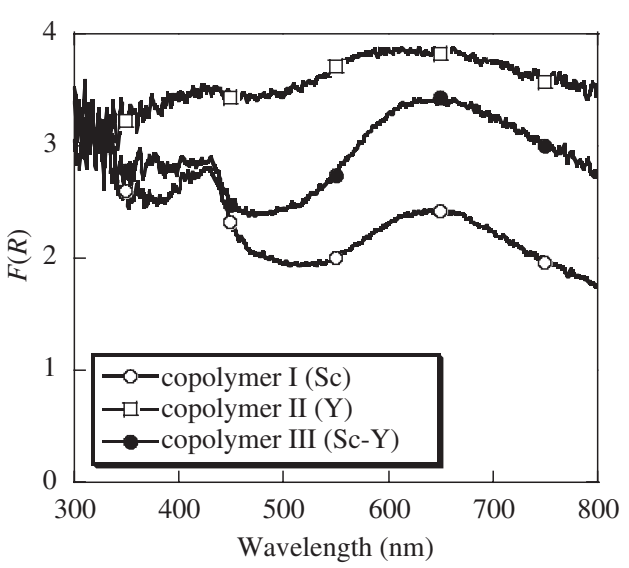

Figure 4. The UV-vis spectra of the hybrid copolymers. The spectra were taken by using the diffusion reflecting method. $F(R)$ is a coefficient of diffusion reflecting which is calculated by the following equation: $F(R)=\{1-R(\lambda)\}^{2} / 2 R(\lambda)$, where $R(\lambda)$ is the reflection rate of the sample.

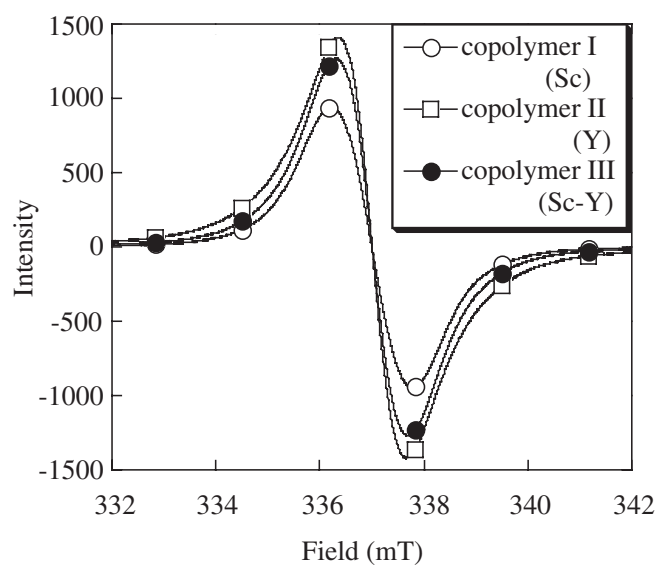

Figure 5. The ESR spectra of the hybrid copolymers. The spectra of the copolymers were taken using $5 \mathrm{mg}$ of sample by Joel JES-TE 200. The measurement conditions: C. Field, 337.00 $\mathrm{mT}$; Power, $10.00 \mu \mathrm{W}$; Sweep Width, $5 \mathrm{mT}$; Modulation Width, $0.5 \mathrm{mT}$; Time Constant, $0.1 \mathrm{~S}$; Temp., room temperature.

tively. These findings indicate that the ability of electron transfer was largre in the order of II $>$ III $>$ I.

In order to evaluate the electron transfer process of the copolymers theoretically, ab-initio calculations were performed using copolymer models shown in Figure 6, and the results of RHF/STO-3G calculations $^{7}$ were summarized in Table III. In models 1 (Sc) and $2(\mathrm{Y})$, HOMO belong to the phenylene group and LUMO belong to the metal atoms (Figures 7 and 8), and, in model 3 (Sc-Y), HOMO belong to the phenylene group and LUMO belong to the yttrium atom (Figure 9). These results indicate that the electron transfer from the phenylene group to the metal atoms takes place to form a cation radical on the phenylene group. In model $3(\mathrm{Sc}-\mathrm{Y}$ ), it was found that $\mathrm{LUMO}+1$ belong to the scandium atom, and that 
Table III. The HOMO/LUMO energy levels of the copolymer models ${ }^{\mathrm{a}}$

\begin{tabular}{ccccccc}
\hline Model & Metal & HOMO & LUMO & LUMO +1 & $\Delta E^{\mathrm{b}}$ & $\Delta E+1^{\mathrm{c}}$ \\
\hline 1 & Sc & -5.551 & 3.157 & - & 8.727 & - \\
2 & $\mathrm{Y}$ & -5.197 & 2.095 & - & 7.292 & - \\
3 & Sc-Y & -5.279 & 2.041 & 3.347 & 7.320 & 8.626 \\
\hline
\end{tabular}

${ }^{\mathrm{a}}$ The dimension of energy level is $\mathrm{eV} .{ }^{\mathrm{b}} \Delta E$ is the value of HOMO/LUMO energy difference. ${ }^{\mathrm{c}} \Delta E+1$ is the value of $\mathrm{HOMO} / \mathrm{LUMO}+1$ energy difference.

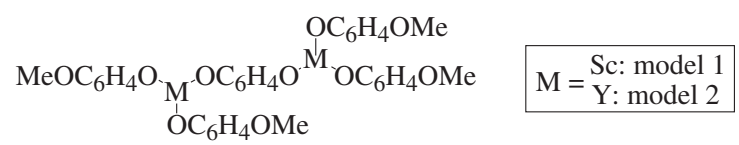

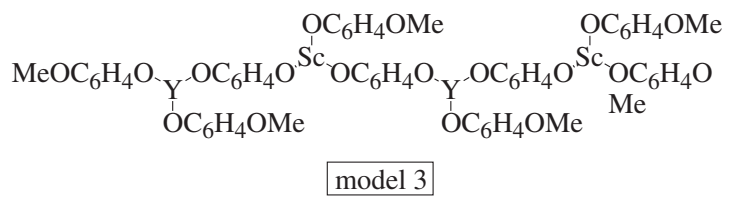

Figure 6. The calculation models.

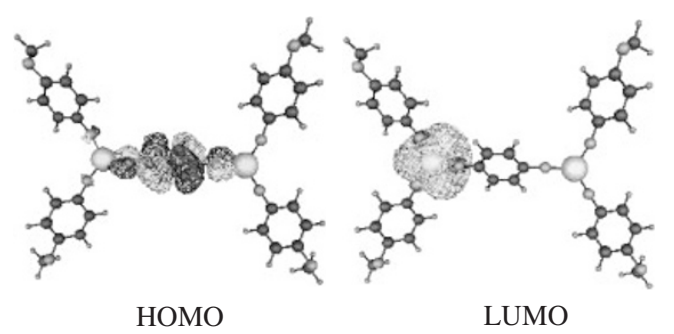

Figure 7. HOMO/LUMO distribution of the model $1(\mathrm{Sc})$.

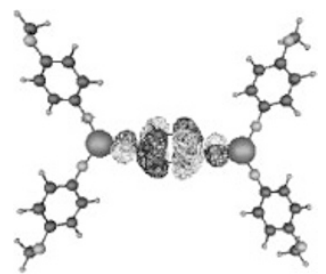

HOMO

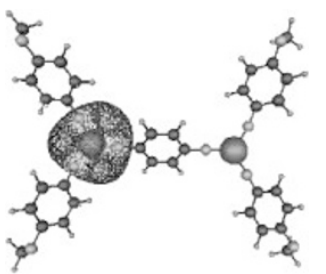

LUMO
Figure 8. HOMO/LUMO distribution of the model $2(\mathrm{Y})$.

the value of the HOMO/LUMO +1 energy difference $(\Delta E+1)$ is $8.626 \mathrm{eV}$. The value of $\Delta E+1(8.626$ $\mathrm{eV})$ in model 3 is not essentially different from the $\Delta E$ value $(8.727 \mathrm{eV})$ of model $1(\mathrm{Sc})$, however, the degree of electron transfer in copolymer III was higher than that in copolymer I as mentioned above, suggesting that yttrium atom effectively assists the electron transfer to scandium atom. On the other hand, although the value of $\Delta E+1(8.626 \mathrm{eV})$ in model 3 is higher than that $(7.292 \mathrm{eV})$ in model 2 , a significant
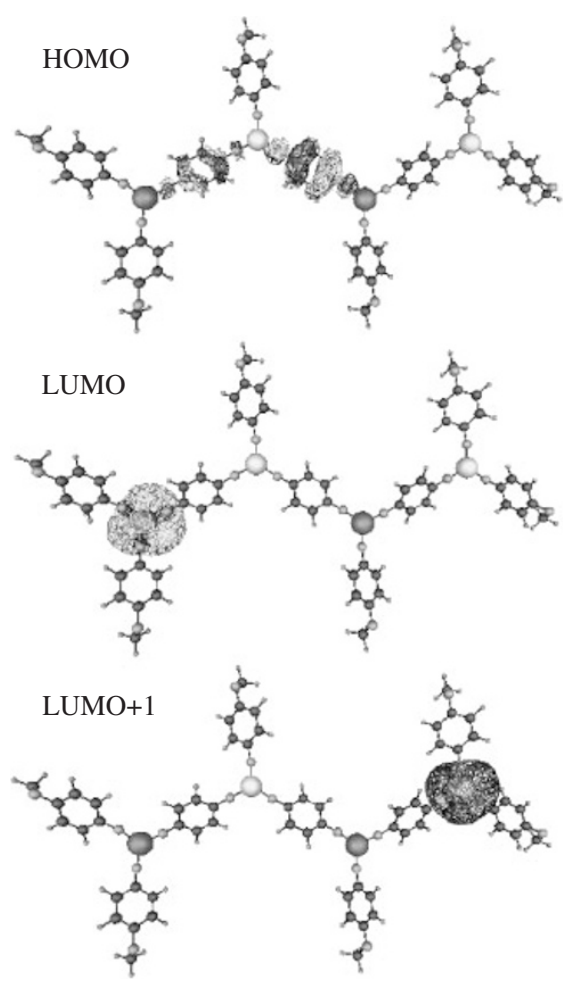

Figure 9. HOMO/LUMO/LUMO +1 distribustion of the model 3 (Sc-Y).

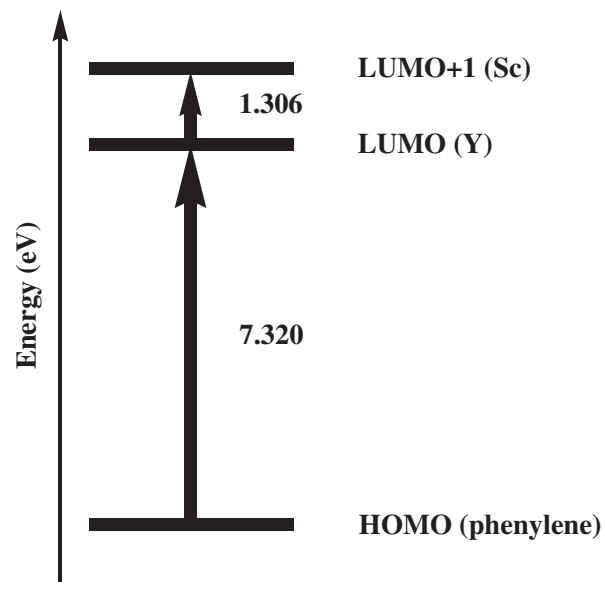

Scheme 2. A two-step electron excitation of the copolymer III $(\mathrm{Sc}-\mathrm{Y})$.

decrease of electron transfer was not detected for copolymer III, suggesting that effective electron transfer and charge separation take place in III. Therefore, the electron transfer of copolymer III ( $\mathrm{Sc}-\mathrm{Y})$ seems to take place initially from the phenylene group to the yttrium atom at a lower energy-level and then to the scandium atom at a higher energy-level. In other words, copolymer III is deduced to have the abilities of effective electron transfer and charge separation via two-step electron excitation (Scheme 2), as seen in a photosynthesis of plants.

We believe that various types of the multi-element 


\section{H. MATSUI et al.}

hybrid polymers will be obtained from the reactions of various metal alkoxides with diols and/or dithiols and also from the reactions of various metal halides with the sodium and/or lithium salts of diols and/or dithiols, and that such materials will be extremely useful in many fields, for example, optical, electronic, and magnetic devices, and photocatalysts.

\section{REFERENCES}

1. K. Seki, S. Takamizawa, and W. Mori, Chem. Lett., 332 (2001).

2. Y. B. Dong, J. Y. Cheng, H. Y. Wang, R. Q. Huang, and
B. Tang, Chem. Mater., 15, 2593 (2003).

3. H. Matsui, A. Okada, and M. Yoshihara, J. Mater. Sci. Lett., 20, 1151 (2001).

4. M. Yoshihara, T. Takeuchi, H. Matsui, and S. Ito, J. Jpn. Soc. Colour Mater., 74, 229 (2001).

5. M. Yoshihara, H. Oie, A. Okada, H. Matsui, and S. Ohshiro, Macromolecules, 35, 2435 (2002).

6. R. Kudo, H. Matsui, and M. Yoshihara, J. Chem. Soc. Jpn., Chem. Indus. Chem., 3, 1051 (2002).

7. M. W. Schimidt, K. Baldridge, J. A. Boatz, S. T. Elbert, M. S. Gordon, J. H. Jensen, S. Koseki, N. Matsunaga, K. A. Nguyen, S. J. Su, T. L. Windus, M. Dupuis, and J. A. Motgomery, Jr., J. Comput. Chem., 14, 1347 (1993). 